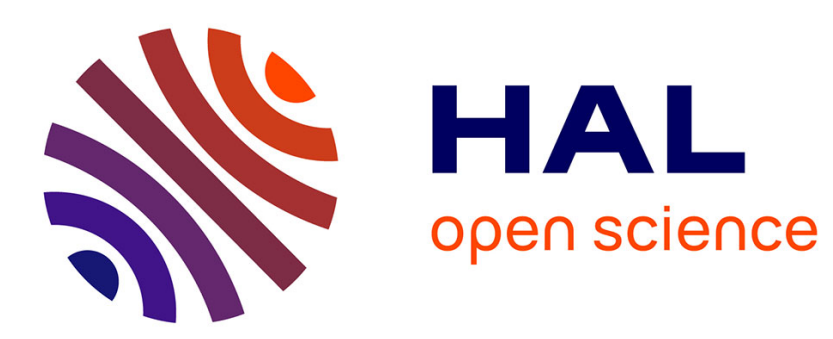

\title{
Phase-change radiative thermal diode
}

Philippe Ben-Abdallah, Svend-Age Biehs

\section{To cite this version:}

Philippe Ben-Abdallah, Svend-Age Biehs. Phase-change radiative thermal diode. Applied Physics Letters, 2013, 103 (19), pp.191907. 10.1063/1.4829618] . hal-01334898

\section{HAL Id: hal-01334898 \\ https://hal-iogs.archives-ouvertes.fr/hal-01334898}

Submitted on 21 Jun 2016

HAL is a multi-disciplinary open access archive for the deposit and dissemination of scientific research documents, whether they are published or not. The documents may come from teaching and research institutions in France or abroad, or from public or private research centers.
L'archive ouverte pluridisciplinaire HAL, est destinée au dépôt et à la diffusion de documents scientifiques de niveau recherche, publiés ou non, émanant des établissements d'enseignement et de recherche français ou étrangers, des laboratoires publics ou privés. 


\title{
Phase-change radiative thermal diode
}

\author{
Philippe Ben-Abdallah ${ }^{1, a)}$ and Svend-Age Biehs ${ }^{2, b)}$ \\ ${ }^{1}$ Laboratoire Charles Fabry, UMR 8501, Institut d'Optique, CNRS, Université Paris-Sud 11, 2 , \\ Avenue Augustin Fresnel, 91127 Palaiseau Cedex, France \\ ${ }^{2}$ Institut für Physik, Carl von Ossietzky Universität, D-26111 Oldenburg, Germany
}

(Received 10 July 2013; accepted 24 October 2013; published online 7 November 2013)

\begin{abstract}
A thermal diode transports heat mainly in one preferential direction rather than in the opposite direction. This behavior is generally due to the non-linear dependence of certain physical properties with respect to the temperature. Here we introduce a radiative thermal diode which rectifies heat transport thanks to the phase transitions of materials. Rectification coefficients greater than $70 \%$ and up to $90 \%$ are shown, even for small temperature differences. This result could have important applications in the development of future contactless thermal circuits or in the conception of radiative coatings for thermal management. (C) 2013 AIP Publishing LLC. [http://dx.doi.org/10.1063/1.4829618]
\end{abstract}

Asymmetry of heat transport with respect to the sign of the temperature gradient between two points is the basic definition of thermal rectification, ${ }^{1,2}$ which is at the heart of a variety of applications as, for example, in thermal regulation, thermal modulation, and heat engines. This unusual thermal behavior has opened the way to new concepts for manipulating the heat flow similar to the electric current in electronic devices. Usually, this manipulation finds its origin in the non-linear behavior of materials with respect to the temperature, which, for the thermal rectification, breaks the symmetry of transfer when the temperature gradient is reversed. The effectiveness of the thermal rectification can be measured by means of the rectification coefficient $\eta=\frac{\left|\Phi_{F}-\Phi_{R}\right|}{\max \left(\Phi_{F}: \Phi_{R}\right)}$, where $\Phi_{F}$ and $\Phi_{R}$ denote the heat flux in the forward and reverse operating mode, respectively. Different solid-state thermal diodes have been conceived during the last decade from various mechanisms (see Ref. 3, for a review on phononic rectification), including nonlinear atomic vibrations, ${ }^{4}$ nonlinearity of the electron gas dispersion relation in metals, ${ }^{5}$ direction dependent Kapitza resistances, ${ }^{6}$ or dependence of the superconducting density of states and phase dependence of heat currents flowing through Josephson junctions. ${ }^{7}$

More Recently, photon-mediated thermal rectifiers ${ }^{8,9}$ have been proposed to tune near-field heat exchange using materials with thermally dependent optical resonances. Since then, numerous mechanisms have been introduced to manipulate the non-radiative heat exchanges ${ }^{10-13}$ between two bodies. Recently, a far-field thermal rectifier has been proposed on the basis of spectrally selective micro or nanostructured thermal emitters ${ }^{14}$ as previously developed to design coherent thermal sources ${ }^{15}$ and to enhance the near-field thermal emission of composite structures. ${ }^{16}$ However, so far only relatively weak radiative and non-radiative thermal rectifications have been highlighted with these mechanisms ( $\eta<44 \%$ in Refs. 8 and 9, $\eta<52 \%$ in Ref. 12 , and $\eta<70 \%$ in Ref. 14 for instance).

In this letter, we propose a radiative thermal rectification principle based on the phase transition of insulator-metal transition (IMT) materials around the operating temperature.

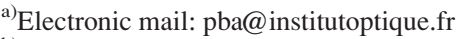

b)Electronic mail: s.age.biehs@uni-oldenburg.de
}

In an IMT material a small smooth change of the temperature around its critical temperature $T_{c}$ causes a sudden qualitative and quantitative change in its optical properties. ${ }^{17}$ In a recent work, van Zwol et al. have shown that the near-field heat-flux exchanged between two media, at close separation distances (subwavelength), could be modulated by several orders of magnitude across the phase transition of vanadium dioxide $\left(\mathrm{VO}_{2}\right)$ deposited on one of the surfaces. ${ }^{11}$ Here the concept of a far-field radiative thermal rectifier is presented. We show that the drastic change in the optical behavior of IMT material across the phase transition between its amorphous and its crystalline phase can lead to a thermal rectification coefficient which is greater than $70 \%$ for small temperature differences and can even be larger than $90 \%$ making these devices serious candidates for thermal diodes without any contact between the high and the low temperature regions

To start, let us consider a system as illustrated in Fig. 1, where two semi-infinite plane bodies, one made of $\mathrm{VO}_{2}$ and

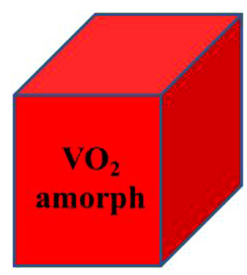

$\mathbf{T}_{\mathrm{L}}=\mathbf{T}_{\mathrm{C}}+\alpha \Delta \mathbf{T}$

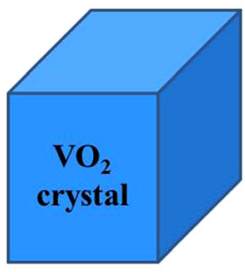

$\mathbf{T}_{\mathbf{L}}=\mathbf{T}_{\mathbf{C}}-\Delta \mathbf{T}$

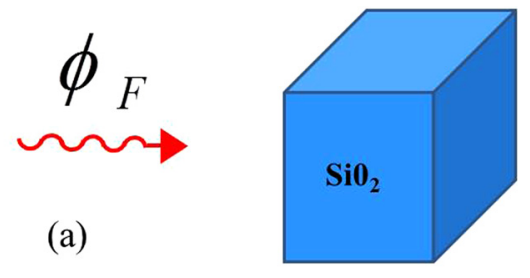

$\mathbf{T}_{\mathbf{R}}=\mathbf{T}_{\mathbf{C}}-\Delta \mathbf{T}$

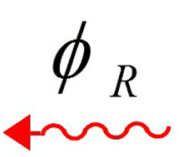

(b)

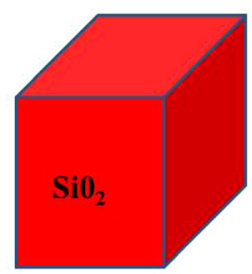

$\mathbf{T}_{\mathbf{R}}=\mathbf{T}_{\mathbf{C}}+\alpha \Delta \mathbf{T}$
FIG. 1. Schematic of a phase-change radiative thermal diode. (a) Forward scenario: the phase-change material is in its amorph state at higher temperature than its transition temperature $T_{c}$. (b) Reverse scenario: the phase change material is in its crystalline state. 
one made of amorphous glass $\left(\mathrm{SiO}_{2}\right)$ at temperatures $T_{\mathrm{L}}$ and $T_{\mathrm{R}}$, respectively. Both media are separated by a vacuum gap of thickness $d$ which is assumed to be much larger than their thermal wavelengths so that heat exchanges are mainly due to propagating photons, i.e., we are considering the far-field regime. We now examine this system in the two following thermal operating modes: (i) In the forward mode $(\mathrm{F})$ the temperature $T_{\mathrm{L}}=T_{c}+\alpha \Delta T$ of $\mathrm{VO}_{2}$ is greater than its critical temperature $T_{c}=340 \mathrm{~K}$ so that $\mathrm{VO}_{2}$ is in its metallic phase while the temperature of the glass medium is $T_{\mathrm{R}}=T_{c}-\Delta T<T_{\mathrm{L}}$. The average temperature can be either positioned at $T_{c}$ (i.e., $\alpha=1$ ) or shifted to lower or higher values. (ii) In the reverse mode (R) $T_{\mathrm{L}}=T_{c}-\Delta T$ so that $\mathrm{VO}_{2}$ is in its crystalline phase and $T_{\mathrm{R}}=T_{c}+\alpha \Delta T>T_{\mathrm{L}}$. In its crystalline (monoclinic) phase, $\mathrm{VO}_{2}$ behaves as a uniaxial medium. Experimental data show that ${ }^{17}$ the optical axis of $\mathrm{VO}_{2}$ films is orthogonal its surface [see Fig. 2 for a plot of the permittivities parallel and perpendicular to the surface]. The net heat flux exchanged per unit surface between two isotropic media or between one uniaxial and one isotropic medium can be written in the general form ${ }^{18-20}$

$$
\begin{aligned}
\Phi_{F / R} & =\int_{0}^{\infty} \frac{\mathrm{d} \omega}{2 \pi} \Delta \Theta(\omega) \sum_{j=\{s, p\}} \int \frac{\mathrm{d}^{2} \boldsymbol{\kappa}}{(2 \pi)^{2}} \mathcal{T}_{j, F / R}(\omega, \boldsymbol{\kappa} ; d), \\
& =\int_{0}^{\infty} \mathrm{d} \omega \Delta \Theta(\omega) \varphi_{F / R}(\omega, d),
\end{aligned}
$$

where $\Delta \Theta(\omega)=\Theta\left(\omega, T_{L}\right)-\Theta\left(\omega, T_{R}\right)$ is the difference of mean energies of Planck oscillators at frequency $\omega$ and at the temperatures of two interacting media. As for $\mathcal{T}_{j, F / R}(\omega, \kappa)$ represents the energy transmission probability carried by the mode $(\omega, \boldsymbol{\kappa})$ ( $\boldsymbol{\kappa}$ is the lateral wave vector) in one of two polarization states (s and p polarization). It is defined for the propagating modes with $\kappa<\omega / c$ by $^{21}$

$$
\mathcal{T}_{j, F / R}(\omega, \kappa ; d)=\operatorname{Tr}\left[\left(\mathbb{1}-\mathbb{R}_{2}^{\dagger} \mathbb{R}_{2}\right) \mathbb{D}^{12}\left(\mathbb{1}-\mathbb{R}_{1}^{\dagger} \mathbb{R}_{1}\right) \mathbb{D}^{12 \dagger}\right]
$$

where the reflection matrix of each interface is given by $(l=1,2)$

$$
\mathbb{R}_{l}=\left[\begin{array}{cc}
r_{l}^{\mathrm{s}, \mathrm{s}}(\omega, \kappa) & r_{l}^{\mathrm{s}, \mathrm{p}}(\omega, \kappa) \\
r_{l}^{\mathrm{p}, \mathrm{s}}(\omega, \kappa) & r_{l}^{\mathrm{p}, \mathrm{p}}(\omega, \kappa)
\end{array}\right]
$$

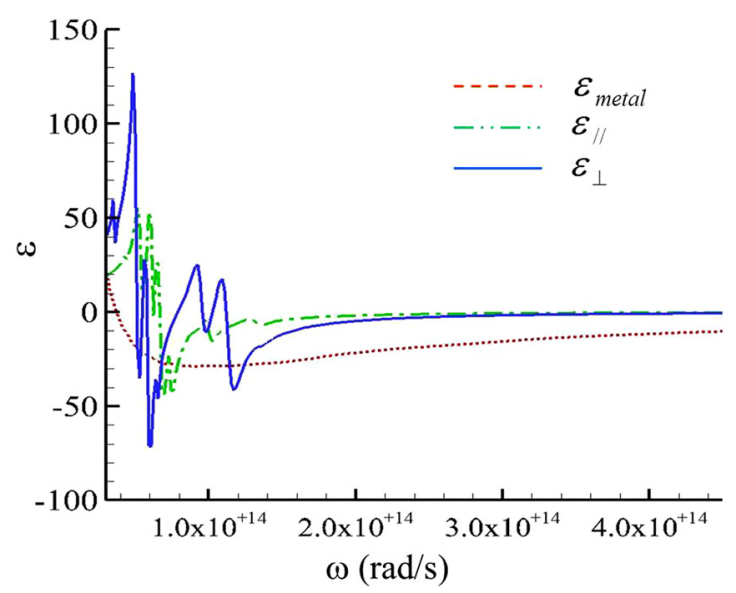

FIG. 2. Permittivities of $\mathrm{VO}_{2}$ in the metallic phase $\left(\epsilon_{\text {metal }}\right)$ and in the crystalline phase $\left(\epsilon_{\|}\right.$and $\left.\epsilon_{\perp}\right)$. and the matrix $\mathbb{D}^{12}$ is defined as

$$
\mathbb{D}^{12}=\left(\mathbb{1}-\mathbb{R}_{1} \mathbb{R}_{2} e^{2 \mathrm{ii} k_{20} d}\right)^{-1},
$$

with $k_{z 0}=\sqrt{\omega^{2} / c^{2}-\kappa^{2}}$. The matrix elements $r_{l}^{j . j^{\prime}}$ of the reflection matrix are the reflection coefficients for the scattering of an incoming $j$-polarized plane wave into an outgoing $j^{\prime}$-polarized wave. For isotropic or uniaxial media with the optical axis orthogonal to the surface $r_{l}^{\mathrm{s}, \mathrm{p}}=r_{l}^{\mathrm{p}, \mathrm{s}}=0$. The remaining reflection coefficients are given by

$$
\begin{gathered}
r_{l}^{\mathrm{s}, \mathrm{s}}=\frac{k_{z 0}-k_{l ; s}}{k_{z 0}+k_{l ; s}}, \\
r_{l}^{\mathrm{p}, \mathrm{p}}=\frac{\epsilon_{\|} k_{z 0}-k_{l ; p}}{\epsilon_{\|} k_{z 0}+k_{l ; p}},
\end{gathered}
$$

where $k_{l ; s, p}$ are solutions of the Fresnel equation

$$
\left(\epsilon_{\|} \frac{\omega^{2}}{c^{2}}-\kappa^{2}-k_{l ; s}^{2}\right)\left(\epsilon_{\|} \epsilon_{\perp} \frac{\omega^{2}}{c^{2}}-\epsilon_{\|} \kappa^{2}-\epsilon_{\perp} k_{l ; p}^{2}\right)=0 .
$$

Here $\epsilon_{\|}$and $\epsilon_{\perp}$ are the permittivities parallel and perpendicular to the surface of the uniaxial material $\mathrm{VO}_{2}$. For amorphous glass which is isotropic we have $\epsilon_{\|}=\epsilon_{\perp}=\epsilon_{\mathrm{SiO}_{2}} .{ }^{22}$ Finally, we simplify the above expressions for the transmission coefficients by taking the limit $d \rightarrow \infty$ and obtain

$$
\mathcal{T}_{j, F / R}(\omega, \kappa)=\frac{\left(1-\left|r_{1}^{\mathrm{j}, \mathrm{j}}(\omega, \kappa)\right|^{2}\right)\left(1-\left|r_{2}^{\mathrm{j}, \mathrm{j}}(\omega, \kappa)\right|^{2}\right)}{1-\left|r_{1}^{\mathrm{j}, \mathrm{j}}(\omega, \kappa)\right|^{2}\left|r_{2}^{\mathrm{j}, \mathrm{j}}(\omega, \kappa)\right|^{2}} .
$$

Figures 3(a) and 3(b) show the flux exchanged in the far field for the forward and the reversed scenario with respect to the temperature difference and the degree of asymmetry of the temperature gradient. The evolution of fluxes $\Phi_{F}$ and $\Phi_{R}$ are very similar (they increase monotonically with the temperature difference). In the forward scenario, the magnitude of the flux is always more than 10 times greater than in the reverse scenario. In order to understand this difference, we have plotted in Fig. 4 the spectral heat flux $\varphi(\omega, d \rightarrow \infty)$ defined in Eq. (1) using the transmission coefficient in Eq. (8) for the forward and reversed scenario. With amorph $\mathrm{VO}_{2}$, the spectral heat flux is broadband and scales like $\propto \omega^{2}$ beyond $\omega=2.2$ $\times 10^{14} \mathrm{rad} / \mathrm{s}$ that is in the frequency range where the diode operates when $T_{\mathrm{L}}>T_{c}$. On the contrary, when $\mathrm{VO}_{2}$ is crystalline, all propagating modes located at frequencies greater than $\omega=1.5 \times 10^{14} \mathrm{rad} / \mathrm{s}$ give a very small and nearly constant contribution to the spectral heat flux and therefore do not transport much energy. This comes from the weak emissivity of $\mathrm{VO}_{2}$ in this state. Hence, $\mathrm{VO}_{2}$ changes for frequencies larger than approximately $\omega=1.5 \times 10^{14} \mathrm{rad} / \mathrm{s}$ from a metallic broadband emitter in the forward scenario to a very strong reflector (and hence very poor thermal emitter) in the reverse configuration. This asymmetry is the key for obtaining a highly efficient phase-change thermal diode.

The rectification coefficient $\eta$ of this rectifier is plotted in Fig. 5. For very small temperature differences we find that $\eta \simeq 70 \%$ illustrating the high efficiency of the phase transition of $\mathrm{VO}_{2}$. This coefficient grows to $\sim 92 \%$ for large temperature differences $\Delta T=200$ and for a large asymmetry 

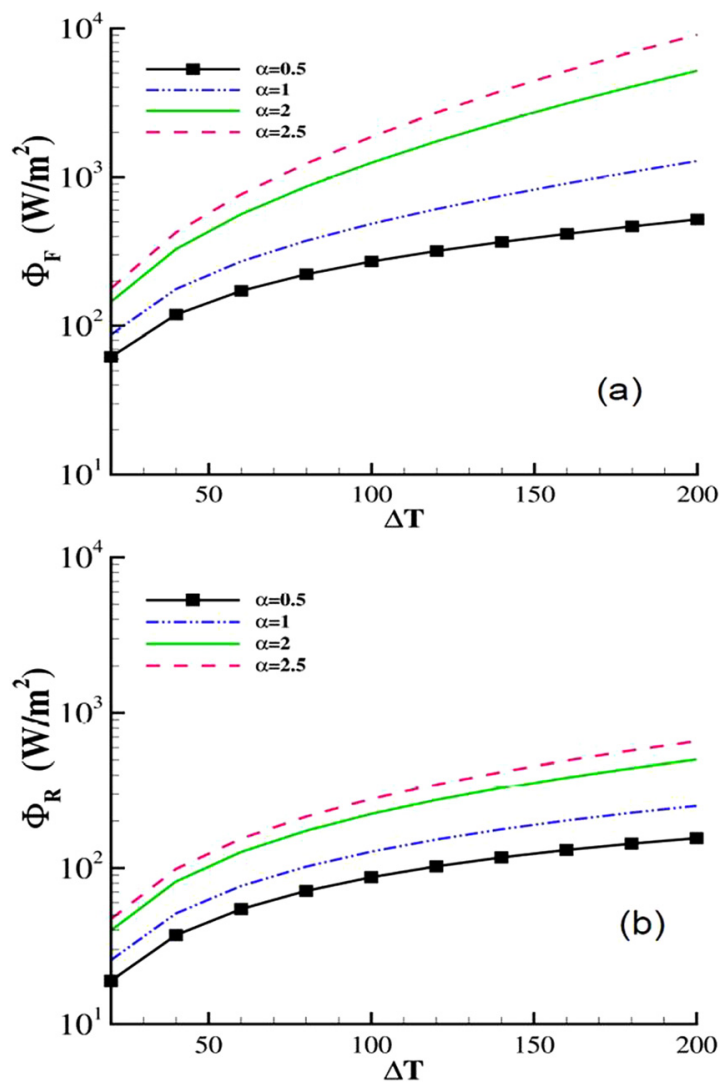

FIG. 3. Orientation dependence of heat flux in (a) the forward and (b) in the reversed temperature scenario between $\mathrm{VO}_{2}$ and glass. The dielectric permittivities of glass and $\mathrm{VO}_{2}$ are taken from Refs. 17 and 22 while the critical transition temperature of $\mathrm{VO}_{2}$ is $T_{c}=340 \mathrm{~K}$.

factor of $\alpha=2.5$. On the contrary, when $\alpha$ is small the thermal rectification does not grow significantly with $\Delta T$. In such a configuration, the distribution function involves the modes of high frequencies in the forward scenario and the modes of low frequency in the reverse scenario. But, according to Fig. 3, the efficiency of heat transport by these modes is relatively high in both cases. As consequence, the flux exchanged in both scenarios is similar as we see in Fig. 2.

In conclusion, we have introduced the concept of a phase-change radiative rectifier. Thanks to the bifurfaction

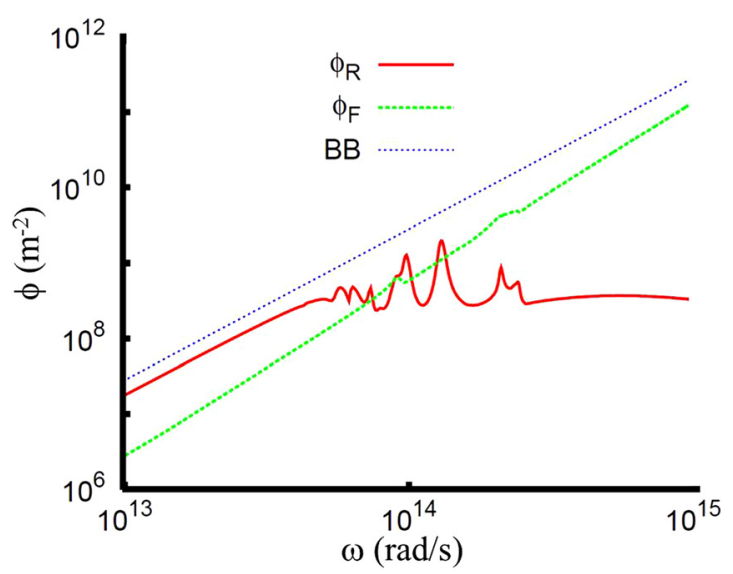

FIG. 4. Plot of the spectral heat flux $\varphi_{F / R}(\omega, d \rightarrow \infty)$ introduced in Eq. (1) as a function of frequency for forward direction where $\mathrm{VO}_{2}$ is metallic and the reverse situation where $\mathrm{VO}_{2}$ is crystalline. In addition, we have plotted $\varphi$ $=\frac{\omega^{2}}{\pi^{2} c^{3}} \frac{c}{4}$ for the case that both materials are perfect black bodies, i.e., $\mathcal{T}_{j, R / F}=1$.

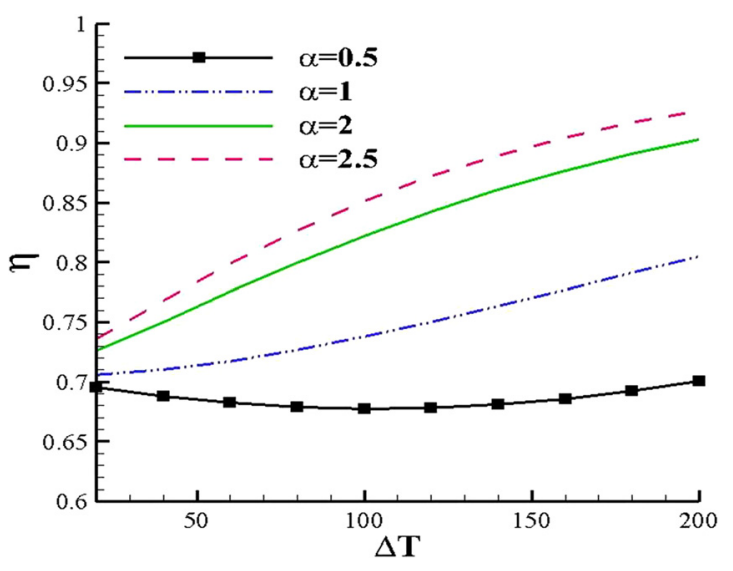

FIG. 5. Rectification coefficient of a $\mathrm{VO}_{2}$-glass system with respect to the temperature discrepancy and the asymmetry of temperature gradient compared with the critical temperature $T_{c}$.

in the optical behavior of MITs close to the critical temperature, large thermal rectification coefficients can be obtained. The here obtained rectification coefficients are between $70 \%$ and $92 \%$ which is rather high as compared to previous radiative rectifier concepts which show rectification coefficients less than $44 \%, 52 \%$, or $70 \% .^{8,9,12,14}$ Hence, by means of using phase change materials, very efficient thermal diodes can be designed. Beyond their potential for thermal management, these radiative thermal rectifiers suggest the possibility to develop out of contact thermal analogs of electronic devices such as radiative thermal transistors and radiative thermal memories for processing information by utilizing photons rather than electrons and thermal sources rather than electric currents.

${ }^{1}$ C. Starr, J. Appl. Phys. 7, 15 (1936).

${ }^{2}$ N. A. Roberts and D. G. Walker, Int. J. Therm. Sci. 50, 648 (2011).

${ }^{3}$ N. Li, J. Ren, L. Wang, G. Zhang, P. Hänggi, and B. Li, Rev. Mod. Phys. 84, 1045-1066 (2012).

${ }^{4}$ B. Li, L. Wang, and G. Casati, Phys. Rev. Lett. 93, 184301 (2004).

${ }^{5}$ D. Segal, Phys. Rev. Lett. 100, 105901 (2008).

${ }^{6}$ H.-Y. Cao, H. Xiang, and X.-G. Gong, Solid State Commun. 152, $1807-1810$ (2012).

${ }^{7}$ M. J. Martinez-Pérez and F. Giazotto, Appl. Phys. Lett.102, 182602 (2013).

${ }^{8}$ C. R. Otey, W. T. Lau, and S. Fan, Phys. Rev. Lett. 104, 154301 (2010).

${ }^{9}$ H. Iizuka and S. Fan, J. Appl. Phys. 112, 024304 (2012).

${ }^{10}$ S.-A. Biehs, F. S. S. Rosa, and P. Ben-Abdallah, Appl. Phys. Lett. 98, 243102 (2011).

${ }^{11}$ P. J. van Zwol, K. Joulain, P. Ben-Abdallah, and J. Chevrier, Phys. Rev. B 84, 161413(R) (2011).

${ }^{12}$ S. Basu and M. Francoeur, Appl. Phys. Lett. 98, 113106 (2011).

${ }^{13}$ L. Zhu, C. R. Otey, and S. Fan, Appl. Phys. Lett. 100, 044104 (2012).

${ }^{14}$ E. Nefzaoui, J. Drevillon, Y. Ezzahri, and K. Joulain, e-print arXiv:1306.6209v1.

${ }^{15}$ J. Drevillon, K. Joulain, P. Ben-Abdallah, and E. Nefzaoui, J. Appl. Phys. 109, 034315 (2011).

${ }^{16} \mathrm{P}$. Ben-Abdallah, K. Joulain, J. Drevillon, and G. Domingues, Appl. Phys. Lett. 94, 153117 (2009).

${ }^{17}$ A. S. Barker, H. W. Verleur, and H. J. Guggenheim, Phys. Rev. Lett. 17, 1286 (1966).

${ }^{18}$ D. Polder and M. Van Hove, Phys. Rev. B 4, 3303 (1971).

${ }^{19}$ S.-A. Biehs, E. Rousseau, and J.-J. Greffet, Phys. Rev. Lett. 105, 234301 (2010).

${ }^{20}$ P. Ben-Abdallah and K. Joulain, Phys. Rev. B 82, 121419(R) (2010).

${ }^{21}$ S.-A. Biehs, P. Ben-Abdallah, F. S. S. Rosa, K. Joulain, and J.-J. Greffet, Opt. Express 19, A1088-A1103 (2011).

${ }^{22}$ Handbook of Optical Constants of Solids, edited by E. Palik (Academic Press, New York, 1998). 